International Journal of Wireless \& Mobile Networks (IJWMN) Vol. 4, No. 3, June 2012

\title{
Node Failures, Data Aggregation Delay AND ITS IMPACT ON POWER OPTIMIZATION IN BALANCED SENSOR NETWORKS
}

\author{
Kamalanathan Kandasamy ${ }^{1}$, Sreedevi A G ${ }^{2}$, P Venkat Rangan ${ }^{3}$ and \\ Maneesha V Ramesh ${ }^{4}$ \\ Amrita Center for Wireless Networks and Applications, \\ Amrita Vishwa Vidyapeetham, Kerala, INDIA \\ ${ }^{1}$ kamalanathan@am.amrita.edu, ${ }^{2}$ agsreedevi@gmail.com, ${ }^{3}$ venkateamrita.edu, \\ ${ }^{4}$ maneesha@am.amrita.edu
}

\begin{abstract}
Wireless Sensor Networks (WSNs) have already emerged as an efficient method to collect data from harsh and human-not-reachable environments. They are employed in many delay-constrained applications where the data collected from the network is analyzed to give timely critical warnings. Such systems need to complete data aggregation and analysis with in minimum time. This research paper is concerned with the design of Multi Level and Two Level Balanced Tree WSN. This research proves that configuration plays a vital role in data aggregation delay, node failure, power optimization and network lifetime when designing a Tree WSN. This paper deals with a method to calculate the overall time for data aggregation in hierarchical network like balanced tree. Our simulation results analyze balanced multi level tree WSN and balanced two level tree WSN on number of computations needed to complete each process and tolerance of node failures. These Balanced configuration leads to less data aggregation delay. The study also deals with an interesting relation between node failure and data aggregation delay in balanced multi level tree WSN.
\end{abstract}

\section{KEYWORDS}

Data Aggregation Delay, Balanced Sensor Network, Maximum Value Computation, Hierarchical Tree Structured Networks, Node Failure, Power Optimization.

\section{INTRODUCTION}

Wireless Sensor Networks hold promise as an effective technology for saving lives when used to monitor, estimate the probability of occurrence, detect, and issue early warnings in most of the applications. Minimizing the data aggregation delay becomes an overwhelming priority for sustained operation of the network. We propose a delay minimization alternative for networks that follow hierarchical tree pattern. The discussed topology includes two level and multi-level balanced sensor network, which can be employed in areas where the network operates in rounds. Each round starts with a computation to identify the maximum value among the sensed data. Following this, all the other sensors, except the maximum valued ones, are switched off for a predetermined duration, after which all of them come back on and the round repeats. The delay consumed by all of the sensors for the max value computation could be minimized by directly minimizing the time taken for the max value computation, algorithms for which are presented in balanced sensor network.

There exists many methods to reduce the data aggregation delays in sensor networks. [6] describes a data aggregation method for tree networks, even though, it enhances the network 
International Journal of Wireless \& Mobile Networks (IJWMN) Vol. 4, No. 3, June 2012

lifetime, it seriously suffers from increased data delivery time. [4], [6] and [7] describe methods of data aggregation, but currently there is no method like the one described in this paper to calculate the delay. This paper provides a simple and totally new mechanism to reduce the delay at the same time a mechanism to calculate the aggregation delay.

Wireless sensor networks promise to allow observation of previously unobservable phenomena consisting of a system of distributed sensors embedded in the physical world. Since these sensor nodes are exposed to harsh and unpredictable environments, sensor network applications must be capable of handling a wide variety of faults such as software errors, node and link failures, and network partitions.

Building reliable and robust sensor network systems is a significant challenge. Also, the faults in sensor networks cannot be approached in the same way as in traditional wired or wireless networks since traditional network protocols are generally not concerned with energy consumption, and they aim to achieve point-to-point reliability. Besides, in sensor networks, the node failures occur much more frequently than in wired, as numerous sensors may exhibit failures due to hardware degradation, inaccurate readings, and environmental changes. It is quite obvious that the node failures decrease the distributed detection performance of the system and data loss.

In this paper, we discuss the different scenarios of node failures that are possible in case of the balanced sensor networks and propose solutions for the same. Node failures are dealt in detail in [1] and [3] but this paper describes a new method to address the same. When a node fails, even though the power consumed by that node can be excluded from the calculation of overall power consumption, the chances of increase in over all power consumption for the whole network and its dependence on the data aggregation delay is also discussed in the paper.

The paper explains data aggregation in applications where lower level sensor nodes perform sensing and transmit data to the corresponding parent. These parents form the intermediate nodes which are capable to perform data aggregation and computation but not sensing. An intermediate node with ' $n$ ' children nodes needs to receive $n$ data and performs its computation. The scenario considered is the time delay needed to compute maximum value from the $n$ received values. To compute the maximum value from $n$ received values, it needs to perform $n$ 1 comparisons, i.e n-1 computations. This is the basic step followed throughout the paper.

The rest of the paper is organized as follows. Section 2 deals with the related literature on this topic. Different levels of balanced sensor network topologies for energy optimization are elucidated in section 3. An analysis of node failure handling strategies for the balanced trees and the proposed solutions are discussed in Section 4. Performance evaluation results obtained for the energy optimization and fault tolerance in balanced sensor network are described in section 5. Finally section 6 concludes the work.

\section{RELATED WORK}

As a part of literature survey, many criteria's to optimize power at different levels were studied. Some duty cycle control algorithms to optimize power in WSNs are described in $[10,11]$. In such networks, when power is conserved, efficiency of the network in computation and prediction is compromised. At the same time [12, 13, 14] discuss topology control algorithms which are hierarchical and location based. This group of networks involve different categories of algorithms each efficiently focussing on the network structure and how the structure reduces power consumption. There is a novel Energy-Aware Data-Centric Routing algorithm for wireless sensor networks explained in [15]. 
International Journal of Wireless \& Mobile Networks (IJWMN) Vol. 4, No. 3, June 2012

Node failures in WSN is a topic less mentioned in the WSN literature, where different issues, solutions and assumptions where brought in. But most of these proposals are for randomly distributed nodes in sensor networks. These approaches are not suitable for WSNs following a tree hierarchy, because in tree hierarchy each level of failures need to be considered differently and solutions differ based on them. Our work in this paper analyses different solutions available for a WSN network, when a node fails.

[13] finds the severity of the node failure by determining its impact on the activity detection accuracy. If the detection accuracy remains above a predefined threshold, a repair is not necessary. If the detection accuracy deteriorates significantly, the system updates by no longer depending on the failed sensor nodes. If the accuracy still remains below the specified threshold, maintenance should be performed.

[1] Analyses the effect of node failures and unreliable communications in a dense sensor network, arranged as a tree of bounded height. This work mainly concentrates on the probability of reliable communication between them to ensure robustness when a node fails.

The technique to identify and recover faults in sensor nodes is analysed in [3] which provides declarative support for modularizing the concerned failure. This work uses the existing macro programming systems for this purpose. [9] addresses node failures in serial network topology by using decision fusion algorithms which are more robust to node failure than the conventional algorithms.

The issue of Quality of Service (QoS) Routing to improve energy consumption in wireless sensor networks is discussed in [16]. This paper formulates the problem of routing sensed information in a WSN network as a path-based energy minimization problem subject to QoS routing constraints expressed in terms of reliability, delay and geo-spatial energy consumption.

The challenging problem of energy minimization for data gathering over a multiple-sources single-sink communication substrate in wireless sensor networks by exploring the energylatency tradeoffs using rate adaptation techniques is studied in [17].

There are some of the limitations of wireless sensor networks as pointed out by Akyildiz et al. [18]. Sensor nodes are densely deployed and are range-limited systems, therefore efficient multi-hop routing algorithms are required. Sensor nodes are unreliable and prone to failure, and the topology of sensor networks changes very frequently, hence it is desirable to set up energy constrained multi-path routing. So we believe that the balanced topology that is discussed in the next section addresses the above issues.

\section{BALANCED SENSOR NETWORK}

\subsection{Two Level Balanced Sensor Network}

In such a network, the total number of sensor nodes is divided equally among the intermediate nodes, and together with the sensors and intermediate nodes they form a 2 level tree hierarchy. Given $\mathrm{N}$ sensors, and $\mathrm{m}$ intermediate nodes, for a balanced network, each intermediate node has $\mathrm{N} / \mathrm{m}$ sensors.

The total energy consumed by the gateway node is proportional to the number of computations and number of transmissions. The total delay or energy consumed or time taken by the gateway node to complete the aggregate computation in a Two Level Balanced Network is,

$$
\operatorname{Tmax}=\left(\frac{N}{m}-1\right) T \operatorname{comp}+(m-1) T \operatorname{comp}+2 \text { Tnet }
$$


The different values of $\mathrm{m}$, gives different delay for the network. The minimum delay for the network is obtained when $m=\sqrt{N}$. Then each IN will have $\sqrt{N}$ sensor nodes attached to it. The delay of such a network can be calculated using,

$$
\operatorname{Tmax}=2(\sqrt{N}-1) \text { Tcomp }+2 \text { Tnet }
$$

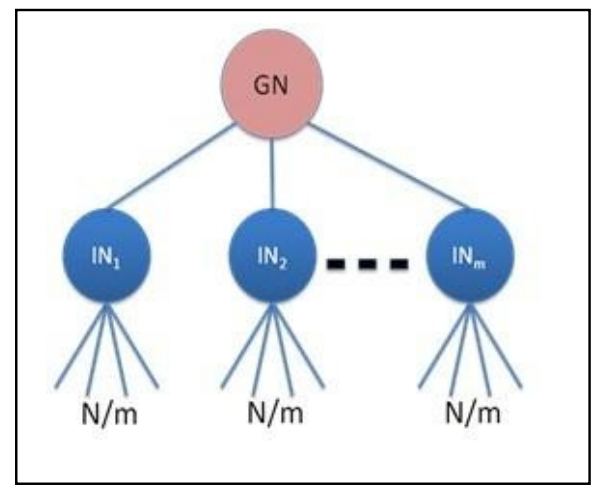

Figure 1: Two Level Balanced Sensor Networks

\subsection{Multi Level Balanced Sensor Network}

We elucidate herein how multi-level balanced sensor network yields minimal delays for completion of computation of sensor aggregates. We consider $\mathrm{N}$ sensor nodes in the network. As figure 1 describes, these $\mathrm{N}$ sensors are equally distributed among some Intermediate Nodes (IN). The results led to the conclusion that, at each height having $\sqrt[H]{N}$, lower-level sensor nodes or IN nodes will yield the aggregation to complete in minimum time.

So, $x_{1}=x_{2}=\cdots=x_{H}=\sqrt[H]{N}$

Here, the data from sensors is passed to the intermediate nodes above it. These intermediate nodes then compute the maximum from it, and this is passed to the IN nodes above it. Likewise, the data moves and ultimately reaches the gateway node where the computation of maximum is performed.

To construct a multi-level balanced network, first step is to compute $N_{\text {one }}=\left(1+\sqrt{r_{n c}}\right)^{2}$ where $r_{n c}=\frac{\text { Tnet }}{\text { Tcomp }}$.

If $N>N_{\text {one }}$, we go for multi-level balanced sensor network, else single level network with all sensor nodes attached to gateway will yield minimum delay in completing the computation. Starting from $N_{\text {one }}+1$ sensors, a two or higher level network yields the least delay. The optimal network (with respect to time taken by max computation) will have Hopt levels, obtained by solving the equation:

$$
N^{\frac{1}{H_{o p t}}}\left(1-\frac{\log N}{H_{o p t}}\right)=1-r_{n c}
$$

Tnet is the time for network communication from one node to another and Tcomp is the time taken at the nodes for comparing two received values to determine their maximum. 
International Journal of Wireless \& Mobile Networks (IJWMN) Vol. 4, No. 3, June 2012

An $\mathrm{H}$ level network of $\mathrm{N}$ sensor nodes will have the following least delay for maximum computation:

$$
\begin{aligned}
T_{\max } & =H T_{\text {net }}+\left(x_{1}-1\right) T_{\text {comp }}+\left(x_{2}-1\right) T_{\text {comp }}+\cdots+\left(x_{H}-1\right) T_{\text {comp }} \\
\text { i.e. } \quad T_{\max } & =H(\sqrt[H]{N}-1) T_{\text {comp }}+H T_{\text {net }}
\end{aligned}
$$

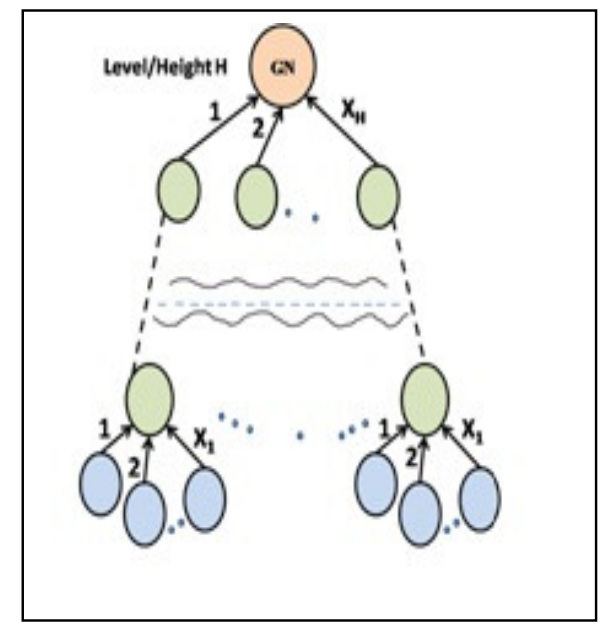

Figure 2: Multi-level Balanced Sensor Network

\section{NODE FAILURE}

Intermediate nodes are nodes between the gateway and lower level sensor node. They are capable of aggregating data and performing computations. They have no sensing capability. At the same time lower level sensor nodes contain sensors which will sense and send the sensed parameter to the intermediate nodes. The chance of nodes getting failed is more. The failure of such nodes results in data loss from lower level nodes to the destination gateway. Since data from those nodes are important for the network, we propose a self reorganisation algorithm.

When a node fails, the gateway node initiates a self reorganization algorithm. This algorithm will be capable of distributing the failed nodes children to the other working nodes. Such an algorithm will be capable of preserving the balanced tree structure that existed before failure. For example, the $\mathrm{N}$ sensor nodes distributed among $\mathrm{m}$ intermediate nodes before failure, need to be re-distributed among m-2 intermediate nodes if 2 intermediate nodes are failed. The algorithm designed need to ensure this balanced topology after the re-distribution also.

Below is the discussion of such failures in two level balanced and multi level balanced sensor network. When the above discussed re-distribution algorithm is initiated, the network's data aggregation delay and power consumed is affected. Following section describes how the data aggregation delay can be calculated and its impact on such failures.

\subsection{Node Failure in Two Level Balanced Sensor Network}

Consider a two level balanced network with $\mathrm{N}$ sensor nodes distributed in a balanced way among $\mathrm{m}$ intermediate nodes (IN). Each IN will have $\mathrm{N} / \mathrm{m}$ children. We assume that any extra sensor nodes remaining after the distribution will be added one by one to each IN, starting from last IN to first. This additional sensor node will contribute 1Tcomp additional to the Tmax which is ignored in the current discussion. 


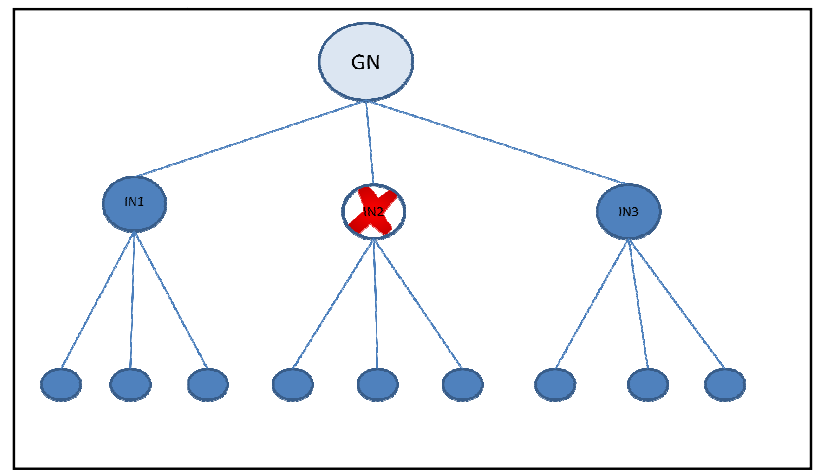

Figure 3 : Before Node Failure

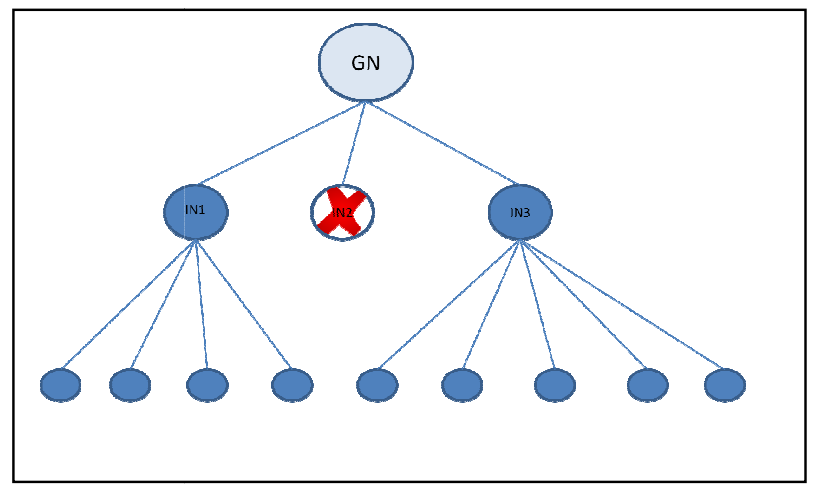

Figure 4: After Node Failure and Reorganization

When an IN node fails, the network executes a self reorganization algorithm. The lower level nodes will organize themselves using the existing IN nodes. For one IN node failure, the network parameters changes as: $\mathrm{N}$ lower level sensor nodes, $\mathrm{m}-1 \mathrm{IN}$ nodes, and $\mathrm{N} /(\mathrm{m}-1)$ lower level nodes to each IN. This new self organisation will increase the number of computations involved at each IN node, there by leading to a small increase in overall energy consumed and aggregation delay.

Consider a balanced network with $\sqrt{N}$ number of Intermediate Nodes and $\frac{N}{\sqrt{N}}$ sensor nodes attached to each IN. Such a network will have,

$$
\operatorname{Tmax}=\left(\frac{N}{\sqrt{N}}-1\right) \operatorname{Tcomp}+(\sqrt{N}-1) \operatorname{Tcomp}+2 \text { Tnet }
$$

When One IN node fails, the new count of IN node will have $(\sqrt{N}-1)$ IN nodes. Now the new distribution will have $\frac{N}{\sqrt{N}-1}$ sensor nodes at each IN.

$$
\operatorname{Tmax}^{\prime}=\left(\frac{N}{\sqrt{N}-1}-1\right) \operatorname{Tcomp}+(\sqrt{N}-1-1) \operatorname{Tcomp}+2 \text { Tnet }
$$

When 'f' nodes fails,

$$
\operatorname{Tmax}^{f}=\left(\frac{N}{\sqrt{N}-f}-1\right) \operatorname{Tcomp}+(\sqrt{N}-f-1) \operatorname{Tcomp}+2 \text { Tnet }
$$


Theorem 1: The energy consumption and data aggregation delay of two level balanced network increases as the number of failures in node increases. This means that the successful operation of the network by tolerating f node failures will compromise the network life time.

Proof: The proof is by contradiction. Let us assume that the number of node failures that the balanced network can tolerate is $\mathrm{f}$, without change in total energy consumed.

The balanced network energy consumption before failure is:

$$
\operatorname{Tmax}=\left(\frac{N}{\sqrt{N}}-1\right) \operatorname{Tcomp}+(\sqrt{N}-1) \text { Tcomp }+2 \text { Tnet }
$$

The balanced network energy consumption after $\mathrm{f}$ failures,

$$
\operatorname{Tmax}^{f}=\left(\frac{N}{\sqrt{N}-f}-1\right) \operatorname{Tcomp}+(\sqrt{N}-f-1) \text { Tcomp }+2 \text { Tnet }
$$

For energy consumption to be the same even before and after failure, Tmax should be equal to Tmax ${ }^{\mathrm{f}}$.

$$
\begin{aligned}
\left(\frac{N}{\sqrt{N}}-1\right)+(\sqrt{N}-1) & =\left(\frac{N}{\sqrt{N}-f}-1\right)+(\sqrt{N}-f-1) \\
\sqrt{N} & =\frac{N}{\sqrt{N}-f}-f \\
\text { i.e } f & =0
\end{aligned}
$$

which contradicts the assumption. So the failures to nodes will increase the number of computations involved, i.e data aggregation delay will increase. As computations increases, the energy consumption will also increase.

\subsection{Node Failure in Multi Level Balanced Sensor Network}

Node failure is a common phenomenon in sensor networks. Intermediate and lower level sensor node failures of multi-level balanced sensor network and its impact on data aggregation delay is discussed in this section. Assuming that one node fails, the gateway node will initialize a self reorganization algorithm, which will redistribute the failed node's children to other existing and working nodes, thereby mitigating the chances of data loss.

When one node at height $\mathrm{h}$ fails, the reorganization of the network needs to equally redistribute $(\sqrt[H]{N})^{h+1}$ nodes at height $\mathrm{h}+1$ among $\left((\sqrt[H]{N})^{h}-1\right)$ nodes at height $\mathrm{h}$, and $\left(\left(\sqrt[H]{N}^{h}-1\right)\right.$ nodes at height $\mathrm{h}$ need to be distributed among $(\sqrt[H]{N})^{h-1}$ nodes at height h-1. For such a network the total delay to complete the data aggregation will change to:

$$
\operatorname{Tmax}=(H-2)(\sqrt[H]{N}-1) T \operatorname{comp}+\left(\frac{(\sqrt[H]{N})^{h+1}}{(\sqrt[H]{N})^{h}-1}-1\right) \operatorname{Tcomp}+\left(\frac{(\sqrt[H]{N})^{h}-1}{(\sqrt[H]{N})^{h-1}}-1\right) \text { Tcomp }+H \text { Tnet }
$$

Similarly, when ' $\mathrm{f}$ ' nodes fail, and all failures occur on the same height $\mathrm{h}$, then the reorganization of the network needs to equally redistribute $(\sqrt[H]{N})^{h+1}$ nodes at height $h+1$ among 
International Journal of Wireless \& Mobile Networks (IJWMN) Vol. 4, No. 3, June 2012

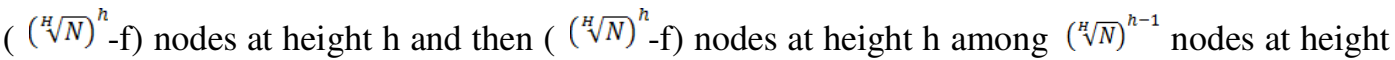
h-1. Thus,

Tmax $=(H-2)(\sqrt[H]{N}-1) T \operatorname{comp}+\left(\frac{(\sqrt[H]{N})^{h+1}}{(\sqrt[H]{N})^{h}-f}-1\right) T \operatorname{comp}+\left(\frac{(\sqrt[H]{N})^{h}-f}{(\sqrt[H]{N})^{h-1}}-1\right)$ Tcomp + H Tnet

When the failed nodes are spread across different heights in a balanced tree, using the same approach discussed above, the time delay for data aggregation can be calculated. Assuming $\mathrm{f}_{1}$ nodes fail at height $h_{1} ; f_{2}$ at height $h_{2} ; \ldots f_{t}$ at height $h_{t}$.

$$
\begin{gathered}
\text { Tmax }=(H-t)(\sqrt[H]{N}-1) T \operatorname{comp}+\sum_{i=1}^{t}\left(\frac{(\sqrt[H]{N})^{h_{i}+1}}{(\sqrt[H]{N})^{h_{i}}-f_{i}}-1\right) \text { Tcomp } \\
+\sum_{i=1}^{t}\left(\frac{(\sqrt[H]{N})^{h_{i}}-f_{i}}{(\sqrt[H]{N})^{h_{i}-1}}-1\right) \text { Tcomp }+H \text { Tnet }
\end{gathered}
$$

where $h_{i}+1$ is less than $H$ for $i=1$ to $t$.

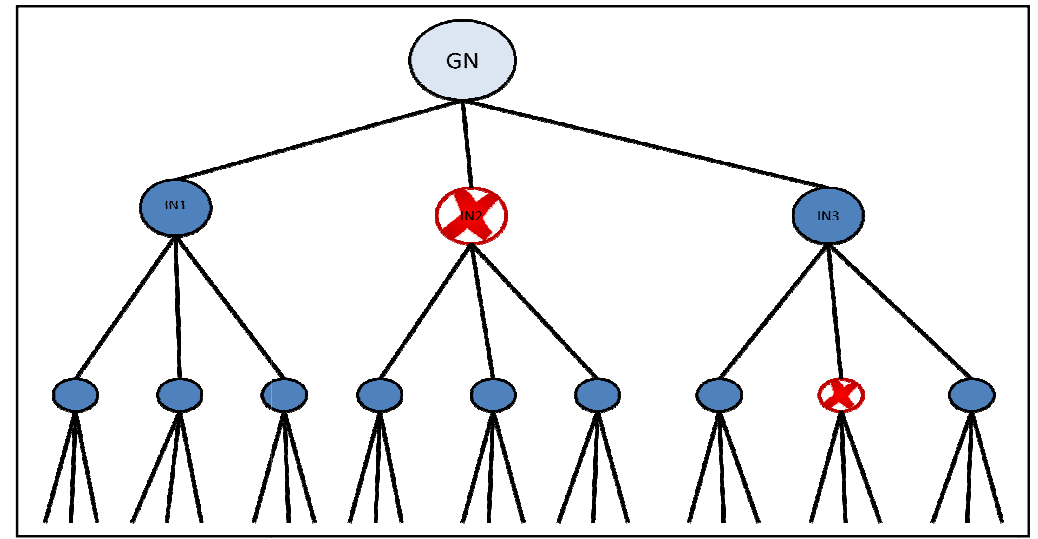

Figure 5: Before Node failures in multi level balanced network

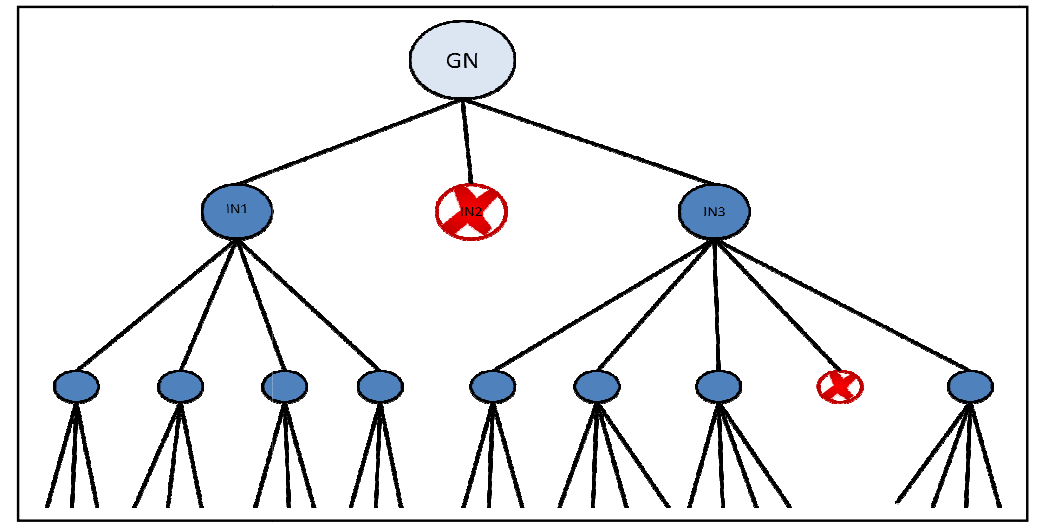

Figure 6: After Self-Reorganization in multi level balanced network

\section{Performance Evaluation}

\subsection{Evaluation Methodology}

To investigate efficiency and validity of the proposed methods, the results will be explained in three sections: first one explains the data aggregation delay of the networks in ideal case, next 
International Journal of Wireless \& Mobile Networks (IJWMN) Vol. 4, No. 3, June 2012

the data aggregation delay when nodes fail in the network, lastly a discussion on impact of these in energy consumed.

The simulation is done in three tools to make the results strong. It includes, the MATLAB which validated the data aggregation delay and its effect on node failure. Secondly, wireless MICAZ motes were used. With the help of nesC programming the tree structure was simulated and its aggregation and energy consumed were tested in the real world. Lastly Qualnet simulation was used to study other performance metrics like throughput, packet loss and average end to end delay. All the results obtained are explained below.

\subsection{Simulation Environment}

In MATLAB, WSN is configured with 100, 500, 1000 and 1500 sensor nodes. The network area considered is square with size of $1000 \times 1000$ for 100 and 500 nodes, and $3000 \times 3000$ for 1000 and 1500 nodes. All the nodes are randomly distributed in the network area. They have the same transmission range of $1500 \mathrm{~m}$ and interference range of $3000 \mathrm{~m}$. The algorithm is carried out for 20 times, the average value of the results is given as the simulation results.

Using the re-organization algorithm, the randomly distributed nodes get attached to intermediate nodes following the tree pattern (i.e in balanced way), then the intermediate nodes to next higher level, same way up to the gateway node. Thus the balanced tree sensor network is created. Now each node will transmit the sensed parameter to its corresponding parent (intermediate node). The intermediate nodes will aggregate the data and will perform the comparisons to obtain the maximum among all the received values. Each intermediate node sends this computed maximum value to its parent, and the process continues until the end result is calculated by the gateway node. The MATLAB results for the data aggregation delay are discussed in next subsection.

MICAZ motes were programmed using nesC language to form the balanced tree structure, and to send data wirelessly as the topology demands. The setup was capable of adapting itself to node failures using the above mentioned method. The aggregation delay was checked for 30 MICAZ motes. The results validated the MATLAB simulation.

QualNet one of the best Wireless Network simulating tool. The simulation was run 30 times on $2000 \times 2000$ terrains, with simulation time 30sec, number of channels 1, channel frequency $2.4 \mathrm{GHz}$ and path loss model two ray model. The main parameter studied included throughput, average end to end delay, and average jitter. The simulation is run for 30 times, and average is taken. The work validated the results obtained and gives an idea about how the system works in the real word environment.

The performance metric considered for evaluation of efficiency of the network includes overall delay to complete the computation in the whole network, power consumed, throughput, average jitter, and average end to end delay.

Overall data aggregation delay: It is the time taken to collect data from lowest level sensor nodes to the completion of computation of the result at the gateway node.

Power Consumption: It is the total power consumed by the network in processing the data as well as for transmitting the computed data.

Average network throughput: It is the average rate of successful message delivery over a communication channel through out the network.

Average jitter: Jitter is a variation in packet transit delay caused by queuing, contention and serialization effects on the path through the network. In general, higher levels of jitter are more likely to occur on either slow or heavily congested links. 
Average end-to-end delay: It is the ratio of total end-to-end delay to the number of packets received where total end-to end delay $+=$ simulation time - data transmit time.

\subsection{Simulation Results}

The MATLAB results obtained are evaluated separately for two level and multi level networks since the height of the network is application dependent. Even though multi level network has less data aggregation delay, it can be chosen only when application demands. As number of nodes in the network increases data aggregation delay of the corresponding network also increases. Figure 7 shows the increase in data aggregation delay with respect to increase in number of nodes. The implementation of the same network in MICAZ motes using nesC validated the result from simulation. The experiment was conducted in 50 MICAZ motes which uses TinyOS. It was really interesting to know that the delay was coinciding with the result from MATLAB.

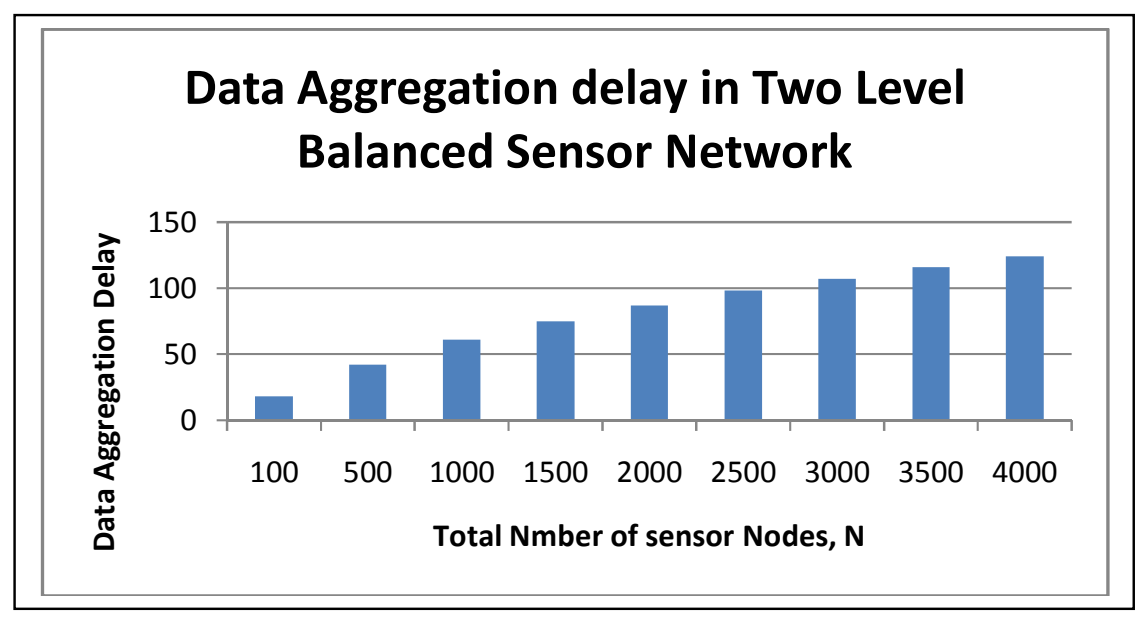

Figure 7: Data aggregation delay in two level balanced sensor networks

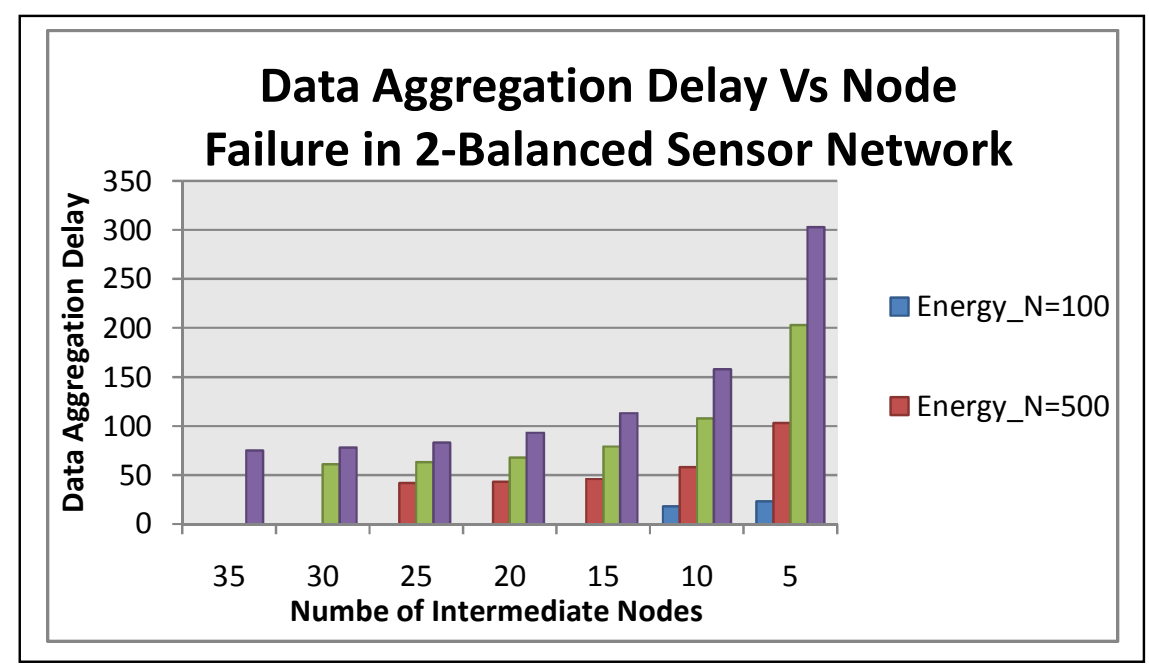

Figure 8: Data aggregation delay Vs node failure

To study the effect of node failure on data aggregation delay in two level network, such a network was simulated on MATLAB. The simulation of node failures shows that, as the node failure increases the over all data aggregation delay of the network increases. This enables us to 
conclude that network life time is compromised as the node failure increases. Figure 8 shows that for each $\mathrm{N}$ value, the energy consumption of the network varies with respect to the number of intermediate nodes the network supports. As node count decreases (which means more and more nodes are failing), the network consumes more aggregation delay and the total lifetime of the network decreases.

The power consumed by a sensor network depends on transmission and computation power. So the consumption will increase as the number of transmission or computation increases. In time critical applications where data aggregation delay is more important with respect to power consumed, one may go for multi level network, where as to optimize power consumption two level network will be suitable. This is because transmission requires more power when compared with computation. In multi level network the number of transmission is more, so will consume more power.

Assume that the network has $100 \%$ lifetime with all nodes working on a two level network with $\mathrm{N}$ total number of sensor nodes and $\mathrm{m}$ intermediate nodes. Let Tmax be the energy consumption with $100 \%$ lifetime. When one node fails, let Tmax' be the new energy consumption. Then the failure of one node will decrease the energy by $100\left(\operatorname{Tmax} /\left(\operatorname{Tmax}^{\prime}\right)-1\right) \%$ and failure of two node will decrease the energy by $100\left(\operatorname{Tmax} /\left(\operatorname{Tmax}^{2}\right)-1\right) \%$ and so on. When $\mathrm{f}$ nodes fails, the energy depletion of the network is by $100\left(\operatorname{Tmax} /\left(\operatorname{Tmax}^{\mathrm{f}}\right)-1\right) \%$. This can be concluded that the energy depletion with respect to number of node failures is dependent on $\mathrm{N}, \mathrm{m}$ and $\mathrm{f}$ values of the network. With respect to its original lifetime, the network is assumed to tolerate $\alpha \%$ lifetime decrease which depends on the application and its lifetime requirement.

The Figure 9 shows how many failures of IN node each N-network will tolerate, assuming different values for $\alpha$. The results show that for existence of the network with good lifetime, the number of tolerable failures need to be less. As failures increases, lifetime decreases.

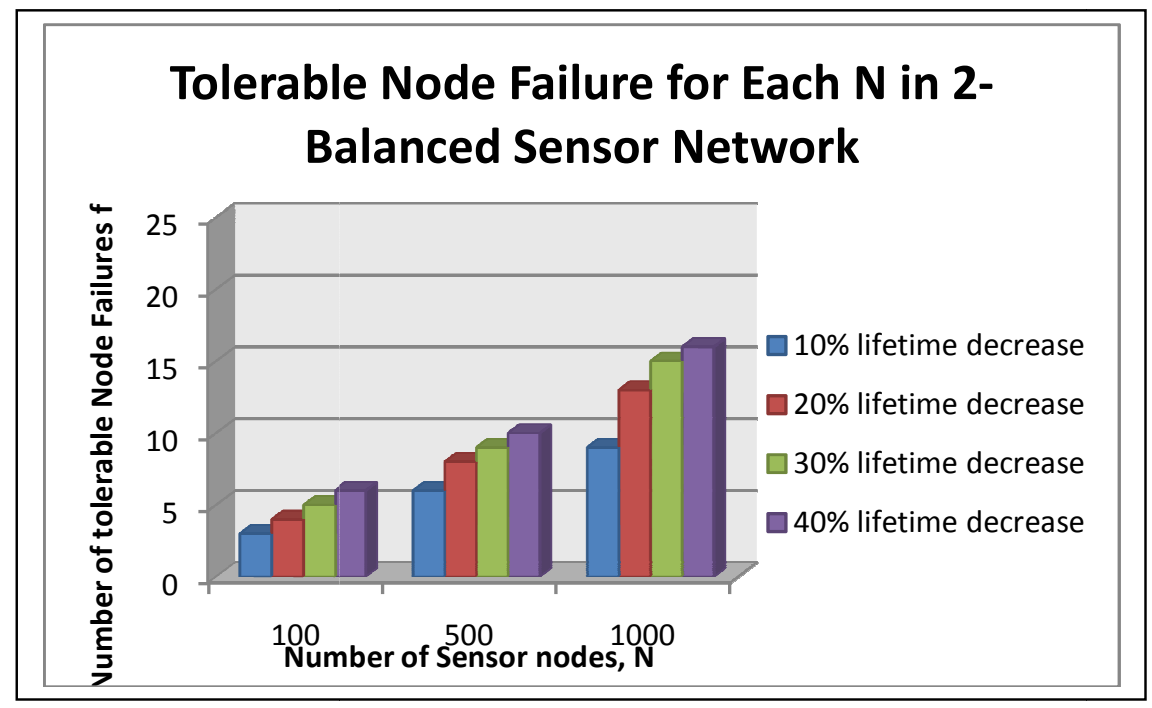

Figure 9: Maximum tolerable node failure for each $\mathrm{N}$

The simulation of multi-level balanced sensor network showing the delay incurred in data aggregation to complete at the gateway node for different values of $\mathrm{N}$ is shown in figure 10 . The figure 10 proves the efficiency of multi-level progressive sensor network, as it completes data 
aggregation with less delay, which proves its efficiency to be used in applications that need to give time critical warnings. The implementation of the same network in MICAZ motes using nesC validated the result from simulation. The experiment was conducted in 50 MICAZ motes which uses TinyOS.

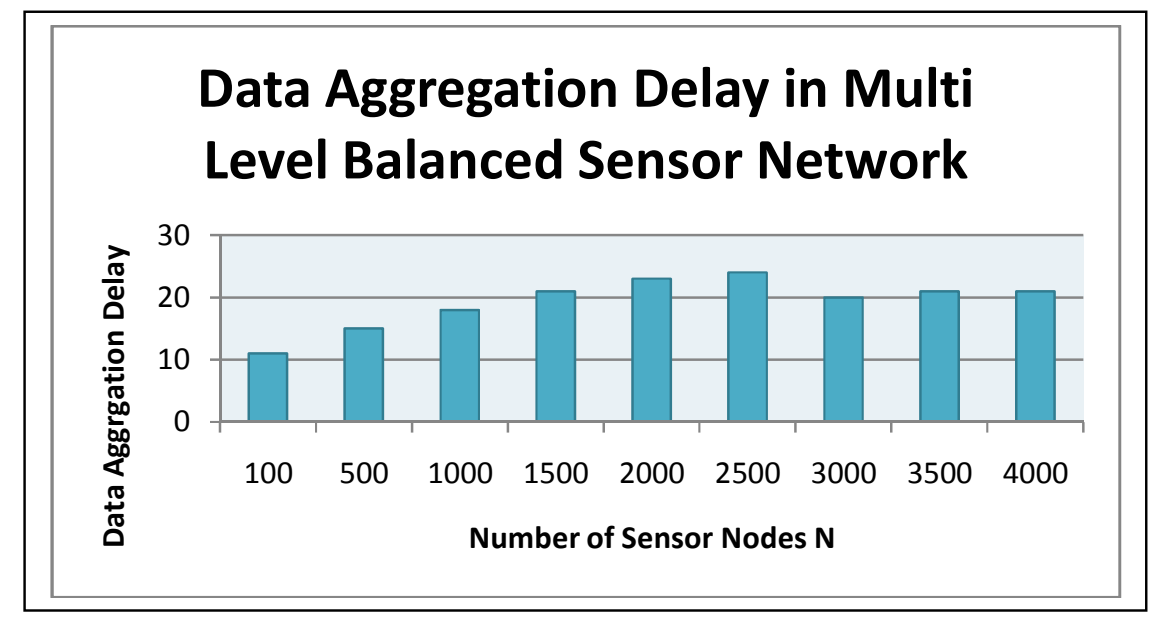

Figure 10: Data Aggregation delay in multi-level balanced sensor network

Figure 11 represents the aggregation delay after network reorganization, for different number of failures at height 2 in multi-level balanced network. The simulation of the same also shows that the delay to complete data aggregation increases as the failures in the network increase.

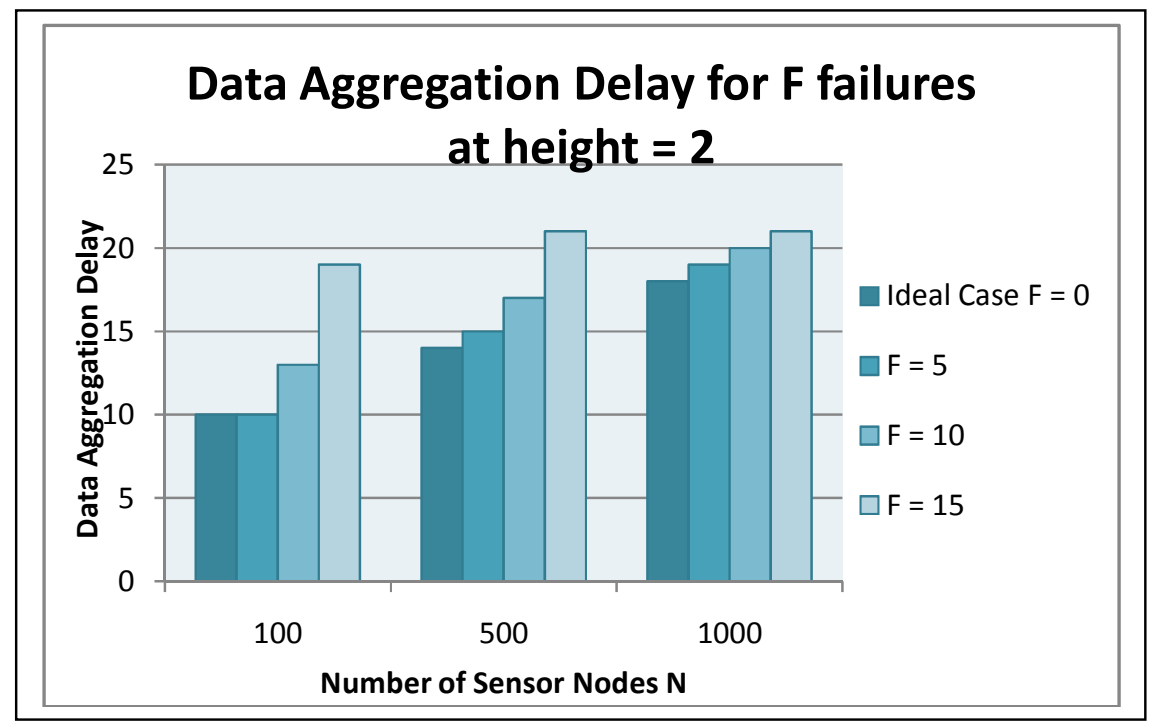

Figure 11: Data Aggregation delay after Reorganization when $\mathrm{f}$ failures occur at height $\mathrm{h}=2$

The simulation of failures at different heights when compared with the failures at same height and ideal case (figure 12) suggests that, when nodes fail at different heights, the delay of the network will increase accordingly. 


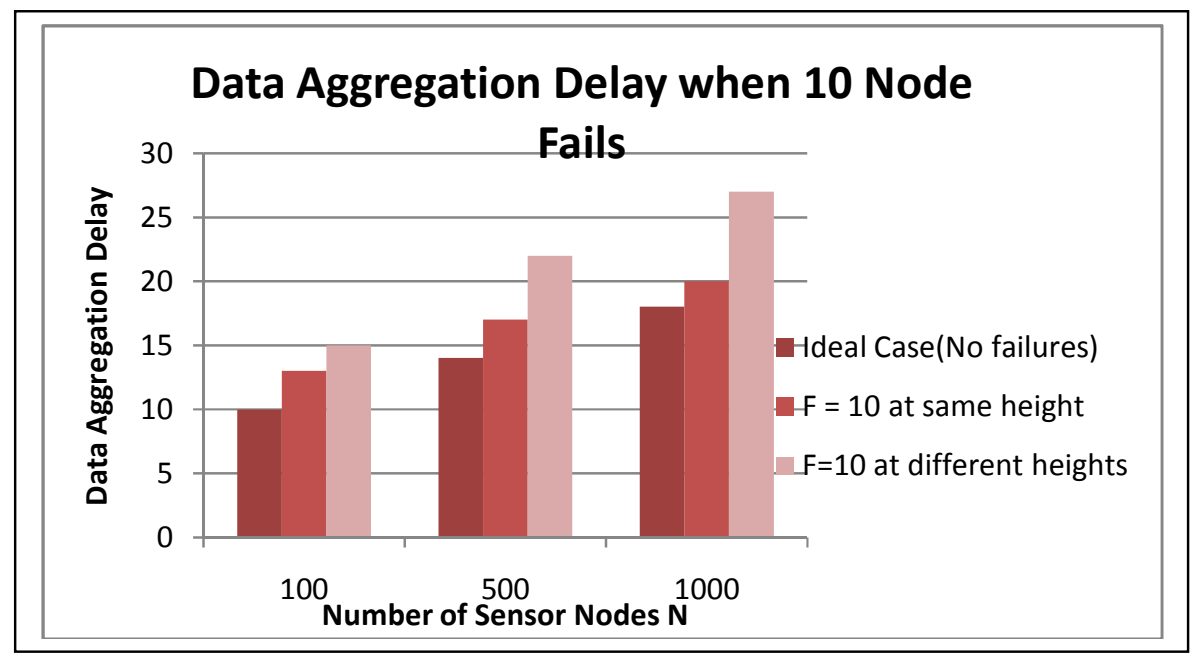

Figure 12: Data Aggregation Delay Comparison when 10 nodes fail at height 2, and 10 nodes fail at different heights (6 nodes at height 3 ; and 4 nodes at height 2 )

The simulation tool QualNet was used to examine the performance parameter like throughput, jitter, and average end-to-end delay. The results obtained are discussed below in detail.

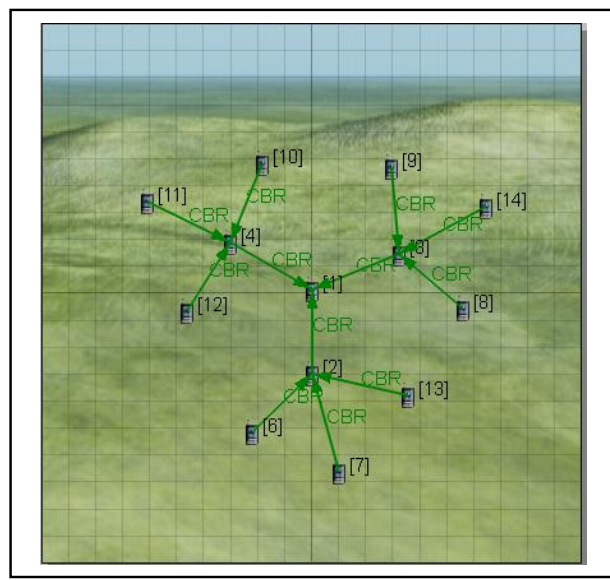

Figure 13: Two Level Balanced Sensor Network

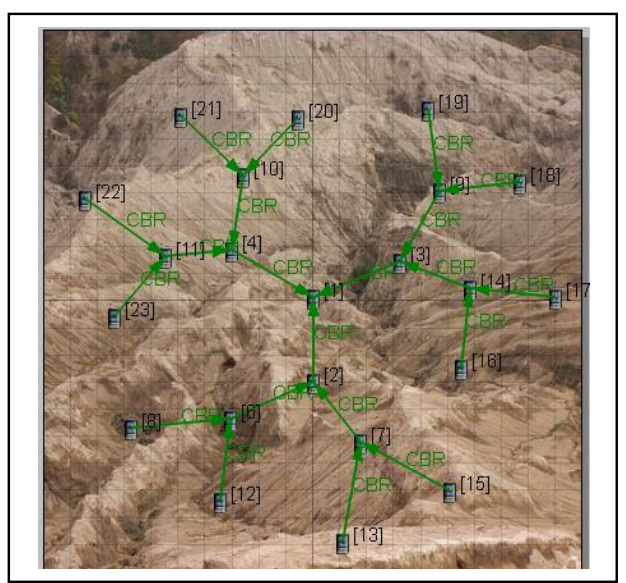

Figure 14: Multi Level Balanced Sensor Network

The network throughput is the average rate of successful message delivery over a communication channel. The simulation of two level and multi level balanced sensor network on QualNet led to the conclusion that two level networks out performs multi level in terms of average throughput (table 1). The average jitter is found to be less in multi level network when compared with the two level balanced sensor networks (table 2). A low jitter is preferred to achieve high range. A preferable low average end-to-end delay is observed in two level balanced sensor network (table 3). 
International Journal of Wireless \& Mobile Networks (IJWMN) Vol. 4, No. 3, June 2012

Table 1: Average throughput for different levels of balanced sensor network

\begin{tabular}{cc}
\hline Network & Average Throughput (bits/sec) \\
\hline Two Level Balanced Sensor Network & 3824 \\
Multi Level Balanced Sensor Network & 3454 \\
\hline
\end{tabular}

Table 2: Average jitter for different levels of balanced sensor network

\begin{tabular}{cc}
\hline Network & Average Jitter (sec) \\
\hline Two Level Balanced Sensor Network & 0.018885906 \\
Multi Level Balanced Sensor Network & 0.012472965 \\
\hline
\end{tabular}

Table 3: Average end-to-end delay for different levels of balanced sensor network

\begin{tabular}{cc}
\hline Network & Average End-to-End Delay (sec) \\
\hline Two Level Balanced Sensor Network & 0.0900268575 \\
Multi Level Balanced Sensor Network & 0.101931549 \\
\hline
\end{tabular}

\section{Conclusions}

In this study, we aimed to calculate the time required from the start of transmission till the maximum value is computed in hierarchical networks such as two level and multi-level of balanced networks.

The paper described a new method to calculate the data aggregation delay in hierarchical tree networks using an example on two level and multi level balanced sensor network. Our results show that, when sensor nodes fail, the gateway initiates a self-reorganization algorithm to redistribute the nodes again to maintain the tree hierarchy, which will increase the data aggregation delay. Moreover, sensor nodes failing at different heights increase the aggregation delay when compared with failures at the same height. Indeed, the lifetime of the network will also get affected as the node failure increases, as energy consumption of the whole network increases.

The future work is planned to focus on the assessment of maximum tolerable failures in the network when given a limit for maximum time delay that the network can take to complete the data aggregation. Then the impact of such data aggregation in energy consumption of hierarchical networks. 
International Journal of Wireless \& Mobile Networks (IJWMN) Vol. 4, No. 3, June 2012

\section{ACKNOWLEDGEMENT}

We would like to express our immense gratitude to our beloved Chancellor Sri (Dr.) Mata Amritanandamayi Devi for providing excellent motivation and inspiration that facilitated this research.

\section{REFERENCES}

[1] Wee Peng Tay, John N. Tsitsiklis, and Moe Z. Win, 'On the Impact of Node Failures and Unreliable Communications in Dense Sensor Networks', IEEE Transactions on Signal Processing, Vol. 56, No. 6, June 2008

[2] S. Ganeriwal, L. K. Balzano, and M. B. Srivastava, 'Reputation-based framework for high integrity sensor networks', ACM Trans. Sen. Netw., June 2008.

[3] Ramakrishna Gummadi Nupur Kothari, Todd Millstein, Ramesh Govindan 'Declarative Failure Recovery for Sensor Networks', AOSD '07 March 12-16, 2007.

[4] Kemal Akkaya and Mohamed Younis, Moustafa Youssef 'Efficient Aggregation of DelayConstrained Data in Wireless Sensor Networks'.

[5] Francisco J. Claudios, Rico Radeke, Dimitri Marandin, Petia Todorova and Slobodanka Tomic, 'Performance Study Of Reconfiguration Algorithms In Cluster-Tree Topologies For Wireless Sensor Networks', The 18th Annual IEEE International Symposium on Personal, Indoor and Mobile Radio Communications.

[6] A.Sivagami, K. Pavai and D. Sridharan, 'Latency Optimized Data Aggregation Timing Model for Wireless Sensor Networks', IJCSI International Journal of Computer Science Issues, Vol. 7, Issue 3, No 6, May 2010

[7] Jianming Zhu, Xiaodong $\mathrm{Hu}$ 'Improved Algorithm For Minimum Data Aggregation Time Problem In Wireless Sensor Networks', Jrl Syst Sci \& Complexity (2008) 21: 626-636.

[8] Krasimira Kapitanova, Enamul Hoque, Jingyuan Li, Daniele Alessandrelli, John A. Stankovic, Sang H. Son, and Kamin Whitehouse ,'Repair Assessment of Sensor Node Failures for Activity Detection'

[9] Mumin Imamoglu, Mehmet Keskinoz, 'Node Failure Handling for Serial Distributed Detection in Wireless Sensor Networks', $21^{\text {st }}$ Annual IEEE International Symposium on Personal, Indoor and Mobile Radio Communications.

[10] M. Yilmaz, "Duty cycle control in wireless sensor networks," A Thesis Submitted to the Graduate School Of Natural And Applied Sciences Of Middle East Technical University, September 2007.

[11] R. K. Rajgopal Kannan and S. S. Iyengar, "Energy and rate based mac protocol for wireless sensor networks," SIGMOD Record, vol. 32, pp. 46 - 55, December 2003.

[12] K. Akkaya and M. Younis, "A survey on routing protocols for wireless sensor networks", Elsevier Ad Hoc Networks 3 (2005) 325-349.

[13] N. H. A. Ababneh, "Energy efficient topology control algorithm for wireless sensor networks," A thesis submitted at The University of Sydney, vol. 1, pp. 1-177, November 2008.

[14] J. W. Fonda, "Energy efficient wireless sensor network protocols for monitoring and prognostics of large scale systems," A Dissertation Presented To The Faculty Of The Graduate School Of The University Of Missouri-Rolla, vol. 1, pp. 10 - 211, 2008. 9999999999999

[15] J. L. Azzedine Boukerche, Xuzhen Cheng, "A performance evaluation of a novel energy-aware data-centric routing algorithm in wireless sensor networks," Springer Science plus Business Media, Inc. Manufactured in The Netherlands, vol. 1, p. 619635, 2005. 1010101010

[16] Antoine B. Bagula and Kuzamunu G. Mazandu F.E. Sandnes et al., "Energy Constrained Multipath Routing in Wireless Sensor Networks”, (Eds.): UIC 2008, LNCS 5061, pp. 453-467, 2008. Springer-Verlag Berlin Heidelberg 2008 
International Journal of Wireless \& Mobile Networks (IJWMN) Vol. 4, No. 3, June 2012

[17] Yang Yu, Viktor K. Prasanna and Bhaskar Krishnamachari, Member, "Energy Minimization for Real-Time Data Gathering in Wireless Sensor Networks", IEEE Transactions on wireless communications, VOL. 5, NO. 11, November 2006

[18] Akyildiz, I.F., Su, W., Sankarasubramaniam, Y., Cayirci, E.: Wireless Sensor Networks: a Survey. Computer Networks 38(4), 393-422 (2002)

\section{Authors}

Kamalanathan Kandasamy is a research associate and a Ph.D. student at Amrita University (Amrita Vishwa Vidyapeetham), Kerala, India. He received B.E. degree in Electronics and Communication Engineering from Bharathiar University, Coimbatore in 1998. His current research interests include cloud computing, energy conservation and security in wireless sensor networks.

Sreedevi A G is a Masters' Student in Wireless Networks and Applications at Amrita University, Kerala, India. Sreedevi received her Bachelor's degree in Computer Science Engineering from Kerala University, India in 2010. Her research interests include wireless sensor networks, adhoc networks, Algorithm analysis and design.
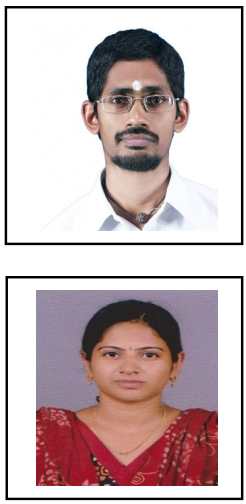

Amrita Vishwa Vidyapeetham is an young and dynamic University established by its Chancellor, Sri Mata Amritanandamayi Devi, popularly called AMMA all over the world and one of the foremost humanitarian leaders of the world today. In 2003, AMMA appointed Dr. P. Venkat Rangan as the Vice Chancellor of Amrita Vishwa Vidyapeetham. Previously, Dr. Rangan founded and directed the Multimedia Laboratory and Internet \& Wireless Networks (WiFi) Research at the University of California, San Diego, (UCSD) where he served as a Professor of

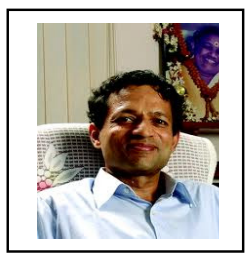
Computer Science and Engineering for 16 years. He is an internationally recognized pioneer of research in Multimedia Systems and Internet E Commerce. In 1993, Dr. Rangan founded the first International Conference on Multimedia: ACM Multimedia 93, for which he was the Program Chairman. This is now the premier world-wide conference on multimedia. Dr. Rangan also founded the first International Journal on Multimedia: ACM/Springer-Verlag Multimedia Systems, which is now the premier journal on Multimedia. Dr. Rangan is also the Fellow of ACM (1998), youngest to achieve this international distinction and he has been awarded with NSF National Young Investigator Award (1993), The NCR Research Innovation Award (1991), and The President of India Gold Medal (1984). In July 2000, Internet World featured Dr. Rangan on its cover page and named him as one of the top 25 Stars of Internet Technologies. Dr. Rangan has over 85 publications in International (mainly IEEE and ACM) Journals and Conferences, and also holds 22 US Patents. 